Research Article

\title{
Prevalence and Determinants of Hypertension Awareness, Treatment, and Control in Botswana: A Nationally Representative Population-Based Survey
}

\author{
Neo M. Tapela $\mathbb{D}^{1,2}$ Lei Clifton, ${ }^{1}$ Gontse Tshisimogo, ${ }^{3}$ Moagi Gaborone, ${ }^{4}$ \\ Tebogo Madidimalo, ${ }^{4}$ Virginia Letsatsi, ${ }^{3}$ Tiny Masupe,${ }^{5}$ Mosepele Mosepele, ${ }^{5}$ \\ Joseph Makhema, ${ }^{2}$ Shahin Lockman, ${ }^{2,6}$ and David J. Hunter ${ }^{1,6}$ \\ ${ }^{1}$ Nuffield Department of Population Health, University of Oxford, Oxford, UK \\ ${ }^{2}$ Botswana Harvard AIDS Institute Partnership, Gaborone, Botswana \\ ${ }^{3}$ National NCD Program, Ministry of Health and Wellness, Gaborone, Botswana \\ ${ }^{4}$ World Health Organization, Gaborone, Botswana \\ ${ }^{5}$ University of Botswana, Gaborone, Botswana \\ ${ }^{6}$ Harvard T.H. Chan School of Public Health, Boston, MA, USA
}

Correspondence should be addressed to Neo M. Tapela; ntapela@gmail.com

Received 17 January 2020; Revised 29 April 2020; Accepted 1 May 2020; Published 31 May 2020

Academic Editor: Salvatore Corrao

Copyright ( $\odot 2020$ Neo M. Tapela et al. This is an open access article distributed under the Creative Commons Attribution License, which permits unrestricted use, distribution, and reproduction in any medium, provided the original work is properly cited.

Introduction. Hypertension is a leading risk factor for cardiovascular mortality and an emerging public health concern in subSaharan Africa. Few studies have examined performance on the management of hypertension in this region, where the context may be distinct from other developing regions. Objectives. We aimed to determine the prevalence and correlates of hypertension, awareness, treatment, and control among adults in Botswana, a middle-income African country undergoing rapid demographic transition and with high HIV burden. Methods. In this 2014 cross-sectional survey of adults aged 15-69 years, information on sociodemographic characteristics, lifestyle behavior, and medical history was collected through in-person interviews and physical measurements (body mass index and triplicate blood pressure (BP)). Hypertension was defined as self-report of use of antihypertensives in the previous two weeks and/or having elevated BP $(\geq 140 / 90 \mathrm{mmHg})$. Multivariable logistic regression was employed to explore factors associated with hypertension, awareness (report of previous diagnosis), treatment (antihypertensives), and control $(\mathrm{BP}<140 / 90)$. Results. Our analysis $(N=4,007)$ yielded an age-standardized hypertension prevalence of $30 \%$ (95\% CI: $28 \%-32 \%, N=1,393)$. Among hypertensives, 54\% (50-58\%) were unaware of their condition, $45 \%$ (40-50\%) of those aware were untreated, and $63 \%(55-70 \%)$ of those on medications were suboptimally treated (BP $\geq 140 / 90 \mathrm{mmHg})$. A fifth of hypertensives who were diagnosed but not on medications had BP $\geq 180 / 110 \mathrm{mmHg}$. Diabetes was the strongest correlate of hypertension and awareness (aOR 4.00, 1.86-8.59; aOR 3.30, 1.44-7.55, respectively). Males were less likely to be aware (aOR 0.62, $0.41-0.94$ ) or controlled (aOR 0.36, 0.16-0.83). Obese individuals were more likely to be treated (aOR 2.17, 1.12-4.22), yet less likely to be controlled (aOR 0.32, 0.15-0.66). Conclusions. We report the first nationally representative estimates of the hypertension care cascade performance in Botswana, which will support planning and future policy evaluations. Findings contribute to the relatively sparse evidence on this subject and may inform development of innovations that improve quality of hypertension management and adherence support in similar settings.

\section{Background}

Cardiovascular diseases (CVD) are the leading cause of mortality worldwide, with $80 \%$ of associated deaths occurring in low- and middle-income countries (LMICs) [1]. Hypertension is the leading risk factor for CVD-related mortality globally contributing to over 7 million premature deaths per year [2]. Addressing hypertension will be 
instrumental to stemming the burden of CVDs and achieving Sustainable Development Goal target 3.4: to reduce premature mortality due to noncommunicable diseases (NCD) by a third by $2030[3,4]$.

Despite this, and the availability of low-cost treatments for hypertension [5], many individuals with the condition living in LMICs are undiagnosed or suboptimally treated (uncontrolled) [5-7]. Studies have previously described high levels of hypertension control in high income countries (HICs), exceeding $80 \%$ among hypertensives on treatment in Canada and the UK $[8,9]$. In contrast, these figures are much lower in LMICs $[10,11]$. While many studies have described determinants of hypertension [10, 12-14], fewer have explored determinants of hypertension awareness, treatment, and control. This is particularly the case for subSaharan Africa, where CVD is the second leading cause of premature mortality [15], prevalence of hypertension among adults ranges from $15 \%$ to $42 \%$ [10], and the context may be distinct from other LMIC settings [16]. Furthermore, many African studies have used data that were not populationbased or not nationally representative [12, 13], and the factors examined have often been limited to sociodemographic and lifestyle behavior.

Given this backdrop, we aimed (a) to determine the prevalence of hypertension awareness, treatment, and control in Botswana, a middle-income African country with a population of 2 million undergoing rapid demographic transition and with high prevalence of HIV (19\%), and (b) to identify correlates of these hypertension care cascade categories among adults, including lifestyle advice and comorbidity. Characterizing gaps in the hypertension care cascade and factors associated with uncontrolled hypertension may inform future research and policy approaches in Botswana and other resource-limited settings with emerging hypertension burden.

\section{Methods}

2.1. Design and Study Population. We conducted secondary analysis of data from a population-based cross-sectional NCD risk factors survey conducted in Botswana between July and December 2014. The survey followed established World Health Organization (WHO) STEPwise NCD risk factor surveillance (STEPS) methodology [17] and employed multistage cluster sampling design to enroll a nationally representative sample of 15-69-year-old citizens residing in the country. The 2011 Population and Housing Census served as the sampling frame, with a population of 2,024,904, residing in 4,845 enumeration areas. Enumeration areas, which correspond to villages in rural areas and neighborhoods in urban areas, were primary sampling units of which a random sample was selected across the 27 health districts, based on probability proportional to size sampling. We note that the selection is not stratified by district. From these, a simple random sample of households (secondary sampling units) was selected, and subsequently a single age-eligible occupant was randomly selected per household using the Kirsch algorithm contained in a hand-held Android device. A complete analysis weight was computed to account for probability of selection at each sampling stage and nonresponse and the differences in age and sex distributions between the sample and Botswana reference 2011 population.

2.2. Data Collection. The survey was implemented by a trained team of 144 Ministry of Health and Wellness (MOH) health providers, supported by the $\mathrm{MOH}$ National Program team and WHO officers. Data were entered directly on to hand-held personal digital assistants, and collection involved three steps: (1) in-person interview, (2) anthropometric (height, weight, and waist circumference) and blood pressure (BP) measurements, and (3) collection of a fasting blood specimen for glucose and lipid profile testing. The interview employed a structured questionnaire, gathering information on participant sociodemographic characteristics, lifestyle behavior, hypertension and other medical history, and prior receipt of advice on lifestyle behavior modification. BP was measured using an automated validated [18] device (OmronM2, Omron Healthcare, Japan) and with three adult cuff sizes available. Following an initial rest of 15 minutes, three $\mathrm{BP}$ measurements were taken at least 5 minutes apart, with the participant in sitting position.

2.3. Definitions. Hypertension was defined as self-report of being on antihypertensive medications in the previous two weeks and/or having elevated BP (mean systolic blood pressure $(\mathrm{SBP}) \geq 140 \mathrm{mmHg}$ or mean diastolic blood pressure (DBP) $\geq 90 \mathrm{mmHg}$ ) during survey measurement. Three further binary hypertension status categories (care cascade phases) were of interest: awareness, treatment, and control. Awareness was defined as report of ever being previously informed of a hypertension diagnosis by a health professional. Treatment was report as being on antihypertensive medications in the previous two weeks. Control was having measured mean SBP $<140$ and mean DBP $<90$. These categories produce mutually exclusive groups of uncontrolled hypertensives previously described in other studies [10, 19-21]: unaware, untreated, or suboptimally treated. Hypertension severity was categorized into three stages following thresholds from the Seventh Report of the Joint National Committee on Prevention, Detection, Evaluation, and Treatment of High Blood Pressure [22]: stage I corresponds to SBP 140-159 mmHg or DBP 90-99 mmHg, stage II SBP $160-179 \mathrm{mmHg}$ or DBP $100-110 \mathrm{mmHg}$, and stage III (also referred to as hypertensive crisis) SBP $>180 \mathrm{mmHg}$ or DBP > $110 \mathrm{mmHg}$.

Variables available in the STEPS dataset that were included in the analysis were sociodemographic characteristics (age, gender, education, and rural residence), known correlates of raised blood pressure and/or risk factors of CVD (alcohol, smoking, added salt, diet, and obesity), and potential correlates of hypertension control that were less often reported (comorbidities, receipt of lifestyle advice). Known diabetes was defined as self-report of being informed by a health professional of having diabetes or of being on glycemic medications in the previous two weeks. Participants were considered having "other comorbidities" if they reported history of asthma, cancer, renal disease, "depression 
or other mental illnesses," or HIV. Specific enquiry was made for the above listed chronic conditions with the exception of HIV, where self-report involved volunteered response to a general question: "do you have any other medical conditions?" (and data entered in free text such as "HIV," "RVD" (retroviral disease), and "on ART" (antiretroviral therapy)).

BMI was computed using the formula of weight divided by height squared $\left(\mathrm{kg} / \mathrm{m}^{2}\right)$. BMI was classified as normal $\left(\leq 25 \mathrm{~kg} / \mathrm{m}^{2}\right)$, overweight $\left(25.1-30 \mathrm{~kg} / \mathrm{m}^{2}\right)$, and obese $\left(>30 \mathrm{~kg} / \mathrm{m}^{2}\right)$. Current smoking was any use of smoked tobacco in the preceding 30 days. Binge use of alcohol was report of drinking six or more units of alcohol in one occasion during the preceding 30 days. Added salt at meals was a response other than "never" (i.e., selecting "rarely," "sometimes," "often," or "always") to the question, "how often do you add salt or salty sauce to your food just before or during eating?" Low fruit or vegetable intake was defined as consuming, on average, less than five servings of fruits or vegetables per day. Participants were considered to have received lifestyle advice if they responded yes to any of the options for the question, "during the past three years, has a doctor or any other health worker advised you to do any of the following: quit using tobacco, reduce salt, eat at least five servings of fruit and/or vegetables each day, start or do more physical activity?" Age groups were categorized to reflect varying CVD risk and conventional demographic groups: 15-29 years, 30-49 years, and 50-69 years. Allocation of rural/urban district status was based on Botswana's 2011 National Population and Housing Census.

2.4. Statistical Analysis. Descriptive analyses were performed, producing proportions for categorical variables and medians/ means for continuous variables. Age- and sex-standardized prevalence estimates for the care cascade phases of hypertension, awareness (among all hypertensives), treatment (among all hypertensives and among those aware), and control (among all hypertensives and among those treated) were computed for the overall population and for subgroups based on predictors of interest. Univariable logistic regression was performed to explore unadjusted relationships between explanatory variables and each hypertension care cascade phase. Multivariable logistic regression models were then fitted to compute fully adjusted odds ratios, with $95 \%$ confidence intervals. All analyses were performed using STATA v15 (Stata Corporation, College Station, Texas, USA) and factoring in study design and weights (svyset and svy commands).

2.5. Ethics. This study was approved by Botswana MOH's Health Research Development Committee. Written informed consent was required for participation in this study. Participants with elevated BP were referred to their local clinic for further review (urgently for those with $\mathrm{BP}>180 / 110$ ).

\section{Results}

A total of 4,070 participants ( $64 \%$ of target sample size), spanning 227 ( $76 \%$ of target) enumeration areas, provided consent and were enrolled in the survey (Tables 1 and 2). Analysis was restricted to participants with nonmissing core variables of gender, age, education, rural residence, hypertension history (medication use, whether previously informed of diagnosis), and measured BP-yielding a final analytic dataset of 4,007 participants (Figures 1 and 2).

3.1. Participant Characteristics. Participant sociodemographic, behavioral and clinical characteristics are summarized in Tables 3 and 4. Participants were young (median age $34(25-47)$ years, $79 \%$ aged $<50$ years) and predominantly female $(2,709,68 \%)$. A third $(1,319,33 \%)$ had primary schooling or less, while $82 \%(3,297)$ resided in rural areas. With respect to cardiovascular risk factors, $38 \%(1,524)$ of participants were overweight or obese, $13 \%$ were current smokers, and 3\% were known diabetics.

3.2. Prevalence of Hypertension, Awareness, Treatment, and Control. We found a high burden of hypertension, with an estimated prevalence of 30\% (95\% CI: $28 \%-32 \%, N=1393$ ) in the general adult population, 58\% (53-64\%) among those aged 50-69 years, and 77\% (66-86\%) among known diabetics (Table 1). We also found large drop-offs in the hypertension care cascade (Figure 1). Over half $(54 \%, 50-58 \%)$ of hypertensives were unaware of their condition, $45 \%(40-50 \%)$ of those who were aware were not on medications, and $63 \%$ (55-70\%) of those treated had BP above target of 140/90 (suboptimally treated). Based on Botswana's 2011 population structure, these figures translate to 218,430 prevalent hypertensives in the country, out of whom 117,724 were unaware, 45,733 were untreated, and 34,575 were suboptimally treated (Figure 1). All told, these results indicate that, out of all prevalent hypertensives, only 9\% (7-12\%) in Botswana are controlled while the remaining $91 \%(88-92 \%)$ are either undiagnosed, untreated, or suboptimally treated (Figure 3).

Table 5 summarizes severity of BP elevation among uncontrolled hypertensives. 8\% (5-12\%) of unaware hypertensives had hypertensive crisis (stage III, BP $>180 / 110$ ) and a further 18\% (14-23\%) had moderately elevated BP (stage II, BP 160-179/100-109). Nearly a fifth of hypertensives who were diagnosed but untreated $(19 \%, 12-28 \%)$ and a fifth of those suboptimally treated $(19 \%, 13-28 \%)$ had hypertensive crisis.

3.3. Factors Associated with Hypertension, Awareness, Treatment and Control. Individuals who were older, less educated, current smokers, obese, or known diabetic were more likely to be hypertensive, based on univariable regression analysis (Table 1). Those who were older, female, or obese were more likely to be aware and to be treated. Having diabetes or other comorbidity was associated with higher likelihood of hypertension awareness. Binge use of alcohol was associated with lower likelihood of awareness and of treatment. Receipt of lifestyle risk factor advice was associated with higher likelihood of awareness and treatment and lower likelihood of hypertension control.

In fully adjusted multivariable logistic regression analysis (Table 2), older age (aOR 1.04, 95\% CI: 1.03-1.06), being male (aOR 1.30, 1.03-1.65), obesity (aOR 2.96, 2.16-4.04), 
TABLE 1: Sociodemographic characteristics of survey participants, by gender (unweighted).

\begin{tabular}{|c|c|c|c|c|c|c|}
\hline \multirow{2}{*}{ Participant characteristic } & \multicolumn{2}{|c|}{ All 4,007 } & \multicolumn{2}{|c|}{ Male } & \multicolumn{2}{|c|}{ Female } \\
\hline & $N$ & $\%$ & $N$ & $\%$ & $N$ & $\%$ \\
\hline \multicolumn{7}{|l|}{ Gender } \\
\hline Female & 2,709 & 67.6 & & & & \\
\hline Male & 1,298 & 32.4 & & & & \\
\hline Missing & 0 & 0.0 & & & & \\
\hline \multicolumn{7}{|l|}{ Age group } \\
\hline $15-29$ years & 1,537 & 38.4 & 554 & 42.7 & 983 & 36.3 \\
\hline $30-49$ years & 1,640 & 40.9 & 509 & 39.2 & 1,131 & 41.8 \\
\hline $50-69$ years & 830 & 20.7 & 235 & 18.1 & 595 & 22.0 \\
\hline Missing & 0 & 0.0 & 0 & 0.0 & 0 & 0.0 \\
\hline Median age in year (IQR) & $34(25-47)$ & & $32(24-45)$ & & $35(26-47)$ & \\
\hline \multicolumn{7}{|l|}{ Marital status } \\
\hline Never married & 2,600 & 64.9 & 874 & 67.3 & 1,726 & 63.7 \\
\hline Married or cohabiting & 1,171 & 29.2 & 381 & 29.4 & 790 & 29.2 \\
\hline Separated, widowed, or divorced & 231 & 5.8 & 42 & 3.2 & 189 & 7.0 \\
\hline Missing & 5 & 0.1 & 1 & 0.1 & 4 & 0.2 \\
\hline \multicolumn{7}{|l|}{ Ethnic group } \\
\hline Motswana & 3,861 & 96.4 & 1,241 & 95.6 & 2,620 & 96.7 \\
\hline Other African & 134 & 3.3 & 52 & 4.0 & 82 & 3.0 \\
\hline European & 2 & 0.1 & 0 & 0.0 & 2 & 0.1 \\
\hline Asian & 3 & 0.1 & 2 & 0.2 & 1 & 0.0 \\
\hline Other & 6 & 0.2 & 2 & 0.2 & 4 & 0.2 \\
\hline Missing & 1 & 0.0 & 1 & 0.1 & 0 & 0.0 \\
\hline \multicolumn{7}{|l|}{ Education } \\
\hline No formal schooling & 373 & 9.3 & 126 & 9.7 & 247 & 9.1 \\
\hline Primary (1-7 years) & 946 & 23.6 & 265 & 20.4 & 681 & 25.1 \\
\hline Secondary (8-12 years) & 1,985 & 49.5 & 624 & 48.1 & 1,361 & 50.2 \\
\hline Tertiary and higher ( $>12$ years) & 703 & 17.5 & 283 & 21.8 & 420 & 15.5 \\
\hline Missing & 0 & 0.0 & 0 & 0.0 & 0 & 0.0 \\
\hline \multicolumn{7}{|l|}{ Employment status } \\
\hline Employed & 1,086 & 27.1 & 465 & 35.8 & 621 & 22.9 \\
\hline Self-employed & 483 & 12.1 & 226 & 17.4 & 257 & 9.5 \\
\hline Unpaid homemaker & 603 & 15.1 & 103 & 7.9 & 500 & 18.5 \\
\hline Student or retired & 513 & 12.8 & 195 & 15.0 & 318 & 11.7 \\
\hline Unemployed & 1,320 & 32.9 & 309 & 23.8 & 1,011 & 37.3 \\
\hline Missing & 2 & 0.1 & 0 & 0.0 & 2 & 0.1 \\
\hline \multicolumn{7}{|l|}{ Rural district } \\
\hline Urban & 710 & 17.7 & 236 & 18.2 & 474 & 17.5 \\
\hline Rural & 3,297 & 82.3 & 1,062 & 81.8 & 2,235 & 82.5 \\
\hline Missing & 0 & 0.0 & 0 & 0.0 & 0 & 0.0 \\
\hline
\end{tabular}

overweight (aOR 1.56, 1.20-2.04), and comorbid diabetes (aOR 4.00, 1.86-8.59) were independent predictors of hypertension. Current smokers tended to be hypertensive; however, this association did not reach statistical significance (aOR 1.40, 0.98-1.99). Men were less likely to be aware (aOR 0.62, 0.41-0.94) and to achieve control (aOR 0.36, $0.16-0.83)$ than women. However, diagnosed men were as likely to receive treatment as women. Being obese was associated with higher likelihood of hypertension awareness (aOR 2.27, 1.40-3.67) and treatment (aOR 2.17, 1.12-4.22), yet lower likelihood of control (aOR 0.32, 0.15-0.66). Comorbid diabetes was the strongest predictor of hypertension awareness (aOR 3.30, 1.44-7.55), with a trend towards higher likelihood of treatment and control although this did not reach statistical significance. Receiving lifestyle risk factor advice in the past three years was strongly associated with higher likelihood of treatment (aOR 4.98, 2.42-10.23) but, paradoxically, lower likelihood of hypertension control $(0.27,0.08-0.83)$. Binge alcohol users were less likely to be treated (aOR 0.41, 0.18-0.94).

\section{Discussion}

We found a prevalence of hypertension that is comparable to other estimates in the region $[10-12,14,23,24]$. Diabetes was the strongest predictor of hypertension, with gender, age, and obesity also associated with hypertension. This provides additional support of findings from other studies describing these predictors of hypertension.

Our results indicate a large antihypertensive treatment gap. In Botswana, it is possible to delink access to medical treatment from financial constraints because of the country's 
TABLE 2: Clinical and behavioral characteristics of survey participants, by gender (unweighted).

\begin{tabular}{|c|c|c|c|c|c|c|}
\hline \multirow{2}{*}{ Participant characteristic } & \multicolumn{2}{|c|}{ All $(4,007)$} & \multicolumn{2}{|c|}{ Male $(N=1,298)$} & \multicolumn{2}{|c|}{ Female $(N=2,709)$} \\
\hline & $N$ & $\%$ & $N$ & $\%$ & $N$ & $\%$ \\
\hline \multicolumn{7}{|l|}{ Body mass index } \\
\hline Underweight, $<18.5$ & 493 & 12.3 & 252 & 19.4 & 241 & 8.9 \\
\hline $\mathrm{Nl}, 18.5 \leq 25$ & 1,881 & 46.9 & 749 & 57.7 & 1,132 & 41.8 \\
\hline Overweight, $25 \leq 30$ & 866 & 21.6 & 216 & 16.6 & 650 & 24.0 \\
\hline Obese, $>30$ & 658 & 16.4 & 79 & 6.1 & 579 & 21.4 \\
\hline Missing & 109 & 2.7 & 2 & 0.2 & 107 & 4.0 \\
\hline \multicolumn{7}{|l|}{ Known diabetes } \\
\hline Not diabetic & 3,898 & 97.3 & 1,268 & 97.7 & 2,630 & 97.1 \\
\hline Diabetic & 109 & 2.7 & 30 & 2.3 & 79 & 2.9 \\
\hline Missing & 0 & 0.0 & 0 & 0.0 & 0 & 0.0 \\
\hline \multicolumn{7}{|c|}{ Other comorbidities (asthma, cancer, renal disease, "depression or other mental illnesses," or HIV) } \\
\hline No & 3,404 & 85.0 & 1,147 & 88.4 & 2,257 & 83.3 \\
\hline Yes & 602 & 15.0 & 151 & 11.6 & 451 & 16.7 \\
\hline Missing & 1 & 0.0 & 0 & 0.0 & 1 & 0.0 \\
\hline \multicolumn{7}{|l|}{ Smoking status } \\
\hline Nonsmoker & 3,491 & 87.1 & 892 & 68.7 & 2,599 & 95.9 \\
\hline Current smoker & 516 & 12.9 & 406 & 31.3 & 110 & 4.1 \\
\hline Missing & 0 & 0.0 & & & 0 & 0.0 \\
\hline \multicolumn{7}{|l|}{ Binge alcohol use } \\
\hline No & 3,459 & 86.3 & 946 & 72.9 & 2,513 & 92.8 \\
\hline Yes & 548 & 13.7 & 352 & 27.1 & 196 & 7.2 \\
\hline Missing & 0 & 0.0 & 0 & 0.0 & 0 & 0.0 \\
\hline \multicolumn{7}{|l|}{ Low fruit/vegetable intake } \\
\hline No & 171 & 4.3 & 42 & 3.2 & 129 & 4.8 \\
\hline Yes & 3,695 & 92.2 & 1,202 & 92.6 & 2,493 & 92.0 \\
\hline Missing & 141 & 3.5 & 54 & 4.2 & 87 & 3.2 \\
\hline \multicolumn{7}{|l|}{ Adding table salt } \\
\hline No & 2,179 & 54.4 & 666 & 51.3 & 1,513 & 55.9 \\
\hline Yes & 1,820 & 45.4 & 630 & 48.5 & 1,190 & 43.9 \\
\hline Missing & 8 & 0.2 & 2 & 0.2 & 6 & 0.2 \\
\hline \multicolumn{7}{|l|}{ Lifestyle advice } \\
\hline No & 1,167 & 29.1 & 484 & 37.3 & 683 & 25.2 \\
\hline Yes & 2,840 & 70.9 & 814 & 62.7 & 2026 & 74.8 \\
\hline Missing & 0 & 0.0 & 0 & 0.0 & 0 & 0.0 \\
\hline
\end{tabular}

Binge use of alcohol was defined as drinking six or more units of alcohol in one occasion during the preceding 30 days. Added salt at meals was defined as a response other than "never" in response to the question, "how often do you add salt or salty sauce to your food just before or during eating?". Other comorbidities: self-reported history asthma, cancer, renal disease, "depression or other mental illnesses," or HIV. The fully adjusted model included all variables listed above but for "diagnosis in previous 12 months" for hypertensives not previously diagnosed. Participants were considered to have received lifestyle advice if they responded yes to any of the options for the question, "during the past three years, has a doctor or any other health worker advised you to do any of the following, quit using tobacco, reduce salt, eat at least five servings of fruit and/or vegetables each day, start or do more physical activity?"

favorable context as a middle-income country with free healthcare for citizens and where over $95 \%$ of the population resides within $8 \mathrm{~km}$ of a health facility [25]. That nearly half of hypertensives who had previously been diagnosed were untreated, and that a fifth of those untreated were in hypertensive crisis $(\mathrm{BP}>180 / 110)$ is sobering. This contradicts the common misperceptions that hypertensives who are untreated have only mildly elevated BP, amenable to lifestyle modification alone rather than management with antihypetensive drugs. This finding also emphasizes the importance of better control of BP given that hypertensive emergency (BP $\geq 180 / 110$ with associated evidence of new or worsening target organ damage) is associated with a oneyear death rate of over $79 \%$ and median survival of 10.4 months if left untreated [26].
The observed treatment gap is likely due to a combination of delivery of poor quality care by providers/health system and limited uptake of available services by patients. Poor quality healthcare, which can be defined as delivery of services that are not compliant with evidence-based guidelines, is a global challenge that contributes to cardiovascular disease-related deaths. In their analysis of the 2016 Global Burden of Disease data, Kruk and colleagues found a toll of 2,358,000 cardiovascular deaths (or $84 \%$ of cardiovascular deaths amenable to healthcare) caused by poorquality care [27]. Urgently needed approaches to address this treatment gap include developing national evidence-based guidelines and training providers on them, strengthening supply chain and facility management, and establishing a pathway for follow-up and reassessment of individuals who 


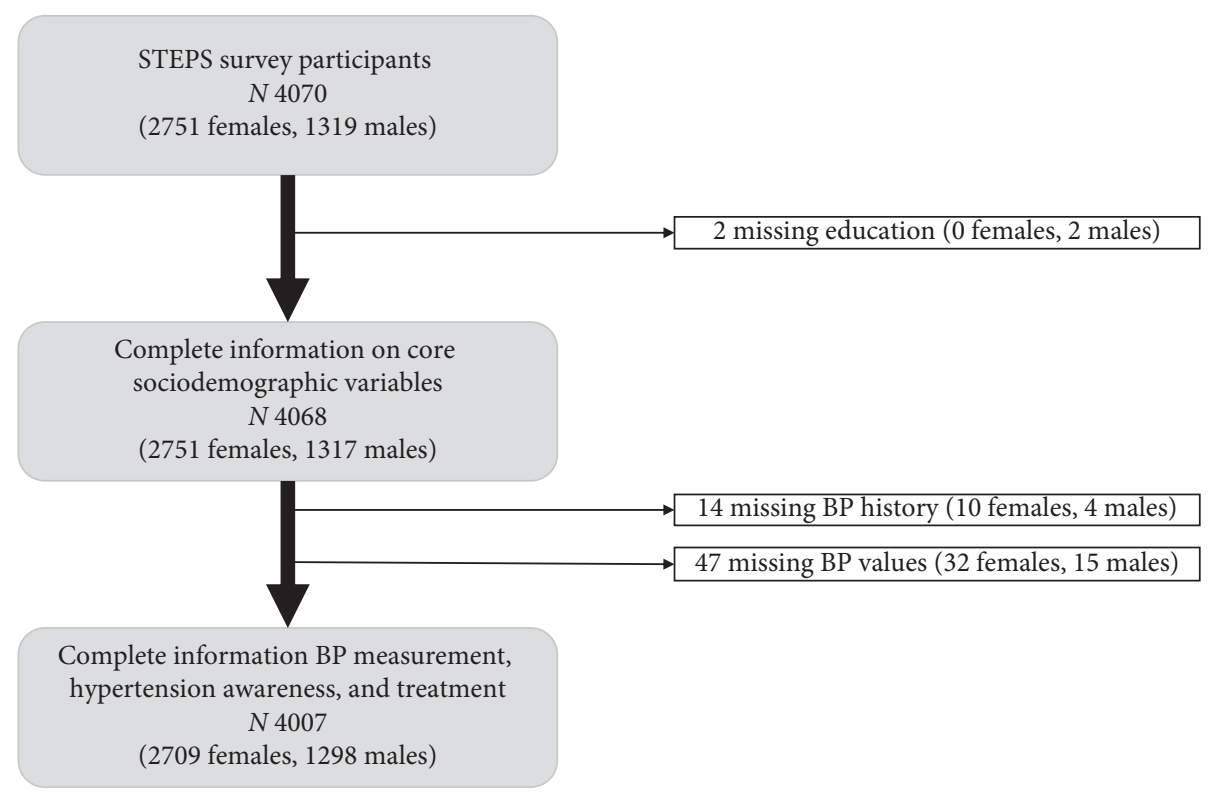

FIgURE 1: Flow chart for selection of analytic dataset.

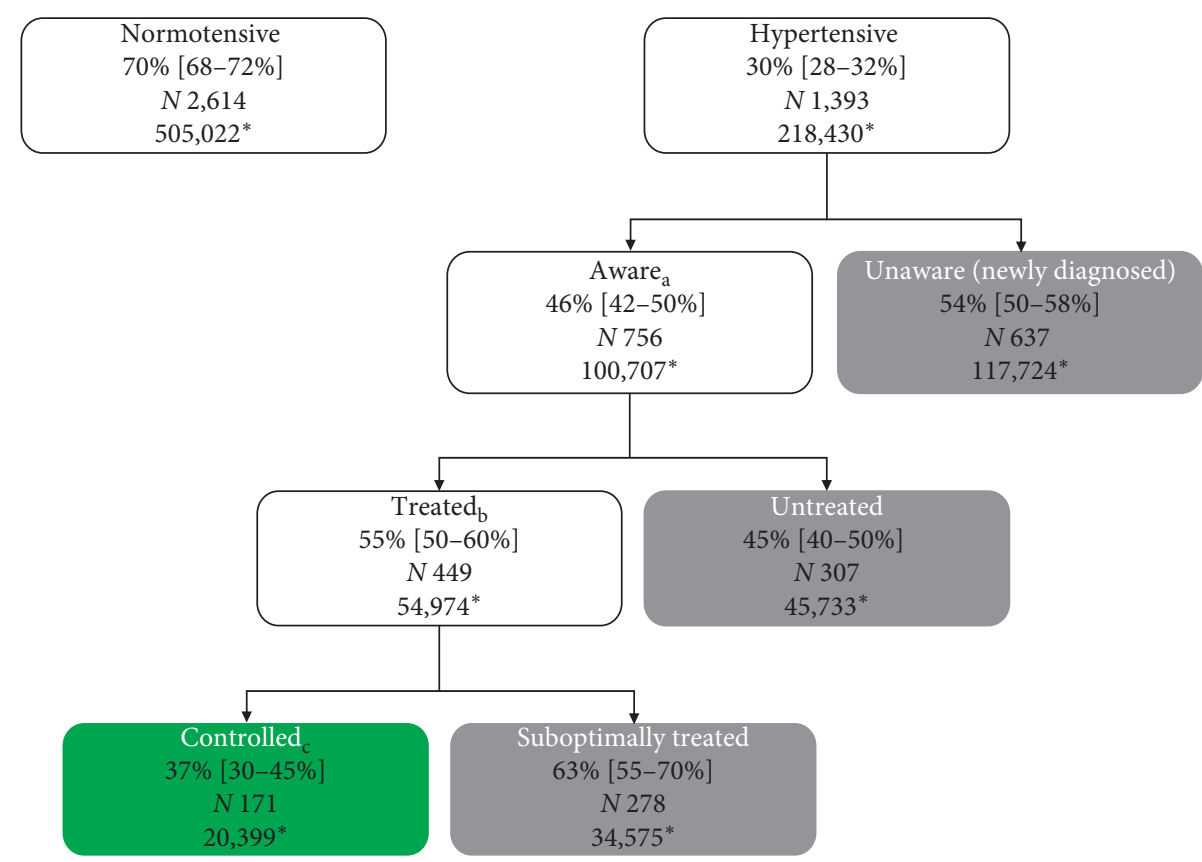

Figure 2: Age-standardized hypertension prevalence, awareness, treatment, and control in the general adult population aged 15-69 years: (a) among participants who are hypertensive; (b) among participants who are aware/diagnosed; (c) among participants who are aware and on medications/treated. Hypertension was defined as self-report of being on antihypertensives in the last two weeks (treated), or having elevated BP based on survey BP measurement (mean SBP $\geq 140 \mathrm{mmHg}$ or mean DBP $\geq 90 \mathrm{mmHg}$ ). Awareness was defined as self-report of being previously informed of hypertension diagnosis by a health professional. Control was defined as measured BP $<140 / 90 .{ }^{*} \mathrm{Number}$ of individuals represented in the category based on Botswana's 2011 Population Census.

previously had only mildly elevated BP. In Botswana, the first evidence-based guidelines for management of hypertension at the primary care level were endorsed in 2016, two years following the data here presented. These were based on WHO's Package of Essential NCDs Interventions (WHO PEN) and contained algorithms for BP screening, CV risk assessment (including glucose and lipid profile), lifestyle advice (smoking cessation, diet, and exercise), and riskbased treatment. Treatment algorithms describe use of antihypertensives for BP persistently $\geq 140 / 90 \mathrm{mmHg}$ (thiazides, +ACE-inhibitors, +calcium channel blockers, and beta-blockers as $1^{\text {st }}, 2^{\text {nd }}, 3^{\text {rd }}$, and $4^{\text {th }}$ line respectively) and use of statins and aspirin if 10 -year CV risk exceeding $30 \%$. We conducted a survey of 142 government health facilities in 
TABLE 3: Distribution of severity of blood pressure elevation among uncontrolled hypertensives (those who are unaware, untreated, and suboptimally treated).

\begin{tabular}{|c|c|c|c|c|c|c|c|c|c|c|c|c|}
\hline \multirow{2}{*}{$\mathrm{BP}$ measurement } & \multicolumn{4}{|c|}{ Unaware $(N=637)$} & \multicolumn{4}{|c|}{ Untreated $(N=307)$} & \multicolumn{4}{|c|}{ Suboptimally treated $(N=278)$} \\
\hline & $\%$ & $95 \%$ & $\mathrm{CI}$ & $N$ & $\%$ & $95 \%$ & $\mathrm{CI}$ & $N$ & $\%$ & $95 \%$ & $\mathrm{CI}$ & $N$ \\
\hline Stage1 & 0.744 & 0.69 & 0.791 & 446 & 0.56 & 0.471 & 0.645 & 168 & 0.529 & 0.444 & 0.6119 & 140 \\
\hline Stage2 BP (S & 0.181 & 0.144 & 0.225 & 139 & 0.253 & 0.193 & 0.325 & 86 & 0.277 & 0.214 & 0.3503 & 89 \\
\hline Stage3 BP $(S B P \geq 180)$ & 0.075 & 0.047 & 0.119 & 52 & 0.187 & 0.121 & 0.279 & 53 & 0.194 & 0.131 & 0.2784 & 49 \\
\hline
\end{tabular}

Unaware hypertensives were those who had elevated BP yet had not previously been informed of a hypertension diagnosis by a health professional. Untreated hypertensives were those who had been previously informed of a hypertension diagnosis but were not on medications in the past 2 weeks and had elevated BP. Suboptimally treated hypertensives were those who had been previously informed of a diagnosis and were on medications yet had elevated BP.

TABLE 4: Prevalence and univariable logistic regression analysis of factors associated with hypertension, awareness, treatment, and control among adults in Botswana.

\begin{tabular}{|c|c|c|c|c|c|c|c|c|c|c|c|c|}
\hline & \multicolumn{6}{|c|}{ Hypertension $(n=1,393)$} & \multicolumn{6}{|c|}{ Awareness, among hypertensives $(n=756)^{\mathrm{a}}$} \\
\hline & $N$ & $\%$ & OR & $95 \%$ & $\mathrm{CI}$ & $p$ & $N$ & $\%$ & OR & $95 \%$ & $\mathrm{CI}$ & $p$ \\
\hline Gender & 4007 & & & & & & 1393 & & & & & \\
\hline Female (ref) & 2709 & $29.0 \%$ & & & & & 963 & $56.6 \%$ & & & & \\
\hline Male & 1298 & $31.3 \%$ & 1.12 & 0.93 & 1.34 & & 430 & $36.7 \%$ & 0.44 & 0.32 & 0.62 & $* * *$ \\
\hline Age group & 4007 & & & & & & 1393 & & & & & \\
\hline $15-29$ years (ref) & 1537 & $19.3 \%$ & & & & & 266 & $24.8 \%$ & & & & \\
\hline $30-49$ years & 1640 & $34.9 \%$ & 2.24 & 1.76 & 2.85 & $* * *$ & 589 & $49.9 \%$ & 3.02 & 1.89 & 4.81 & $* * *$ \\
\hline 50-69 years & 830 & $58.4 \%$ & 5.88 & 4.36 & 7.93 & $* * *$ & 538 & $66.2 \%$ & 5.95 & 3.80 & 9.30 & $* * *$ \\
\hline Highest education & 4007 & & & & & & 1393 & & & & & \\
\hline None (ref) & 373 & $43.6 \%$ & & & & & 198 & $60.2 \%$ & & & & \\
\hline Primary & 946 & $42.2 \%$ & 0.95 & 0.64 & 1.40 & & 465 & $47.3 \%$ & 0.59 & 0.36 & 0.98 & $*$ \\
\hline Secondary & 1985 & $24.5 \%$ & 0.42 & 0.30 & 0.59 & $* * *$ & 521 & $40.4 \%$ & 0.45 & 0.27 & 0.73 & $* *$ \\
\hline Tertiary or higher & 703 & $27.8 \%$ & 0.50 & 0.35 & 0.72 & $* * *$ & 209 & $50.4 \%$ & 0.67 & 0.38 & 1.18 & \\
\hline Residence type & 4007 & & & & & & 1393 & & & & & \\
\hline Urban (ref) & 710 & $27.7 \%$ & & & & & 231 & $49.8 \%$ & & & & \\
\hline Rural & 3297 & $31.1 \%$ & 1.17 & 0.91 & 1.51 & & 1162 & $45.0 \%$ & 0.83 & 0.55 & 1.24 & \\
\hline Current smoking & 4007 & & & & & & 1393 & & & & & \\
\hline No (ref) & 3491 & $28.3 \%$ & & & & & 1201 & $48.8 \%$ & & & & \\
\hline Yes & 516 & $38.6 \%$ & 1.59 & 1.18 & 2.16 & $* *$ & 192 & $37.4 \%$ & 0.63 & 0.38 & 1.04 & \\
\hline Binge alcohol use & 4007 & & & & & & 1393 & & & & & \\
\hline No (ref) & 3459 & $29.2 \%$ & & & & & 1203 & $48.4 \%$ & & & & \\
\hline Yes & 548 & $34.5 \%$ & 1.28 & 0.93 & 1.74 & & 190 & $37.6 \%$ & 0.64 & 0.41 & 1.00 & $*$ \\
\hline Adding table salt & 3999 & & & & & & 1391 & & & & & \\
\hline No (ref) & 2179 & $33.7 \%$ & & & & & 838 & $51.7 \%$ & & & & \\
\hline Yes & 1820 & $26.6 \%$ & 0.71 & 0.58 & 0.87 & & 553 & $38.5 \%$ & 0.58 & 0.41 & 0.83 & $* *$ \\
\hline Body mass index & 3898 & & & & & & 1376 & & & & & \\
\hline Normal, BM I<25 (ref) & 2374 & $24.4 \%$ & & & & & 618 & $33.1 \%$ & & & & \\
\hline Overweight, BMI 25-30 & 866 & $38.6 \%$ & 1.95 & 1.53 & 2.49 & $* * *$ & 379 & $58.3 \%$ & 2.83 & 1.87 & 4.29 & $* * *$ \\
\hline Obese, $\mathrm{BMI}>30$ & 658 & $54.3 \%$ & 3.68 & 2.82 & 4.80 & $* * *$ & 379 & $65.9 \%$ & 3.91 & 2.65 & 5.75 & $* * *$ \\
\hline Known diabetes & 4007 & & & & & & 1393 & & & & & \\
\hline No (ref) & 3898 & $29.2 \%$ & & & & & 1310 & $44.0 \%$ & & & & \\
\hline Yes & 109 & $77.1 \%$ & 8.14 & 4.57 & 14.52 & $* * *$ & 83 & $84.3 \%$ & 6.81 & 2.72 & 17.05 & $* * *$ \\
\hline Other comorbidities & 4006 & & & & & & 1392 & & & & & \\
\hline No (ref) & 3404 & $29.6 \%$ & & & & & 1168 & $43.4 \%$ & & & & \\
\hline Yes & 602 & $33.7 \%$ & 1.21 & 0.89 & 1.64 & & 224 & $61.0 \%$ & 2.04 & 1.34 & 3.11 & $* *$ \\
\hline Lifestyle advice & 4007 & & & & & & 1393 & & & & & \\
\hline No (ref) & 1167 & $27.2 \%$ & & & & & 331 & $20.4 \%$ & & & & \\
\hline \multirow[t]{3}{*}{ Yes } & 2840 & $31.6 \%$ & 1.23 & 0.98 & 1.55 & & 1062 & $56.3 \%$ & 5.01 & 3.37 & 7.46 & $* * *$ \\
\hline & \multicolumn{6}{|c|}{ Treatment, among aware hypertensives $(n=449)^{\mathrm{b}}$} & \multicolumn{6}{|c|}{ Control, among treated hypertensives $(n=171)^{\mathrm{c}}$} \\
\hline & $N$ & $\%$ & OR & $95 \%$ & $\mathrm{CI}$ & $p$ & $N$ & $\%$ & OR & $95 \%$ & CI & $p$ \\
\hline Gender & 756 & & & & & & 449 & & & & & \\
\hline Female (ref) & 585 & $61.2 \%$ & & & & & 363 & $40.6 \%$ & & & & \\
\hline Male & 171 & $45.4 \%$ & 0.53 & 0.33 & 0.84 & $*$ & 86 & $30.7 \%$ & 0.65 & 0.30 & 1.39 & \\
\hline
\end{tabular}


TABLE 4: Continued.

\begin{tabular}{|c|c|c|c|c|c|c|c|c|c|c|c|c|}
\hline & \multicolumn{6}{|c|}{ Hypertension $(n=1,393)$} & \multicolumn{6}{|c|}{ Awareness, among hypertensives $(n=756)^{\mathrm{a}}$} \\
\hline & $N$ & $\%$ & OR & $95 \%$ & $\mathrm{CI}$ & $p$ & $N$ & $\%$ & OR & $95 \%$ & $\mathrm{CI}$ & $p$ \\
\hline Age group & 756 & & & & & & 449 & & & & & \\
\hline $15-29$ years $(\mathrm{ref})$ & 80 & $28.5 \%$ & & & & & 23 & $48.4 \%$ & & & & \\
\hline $30-49$ years & 310 & $50.3 \%$ & 2.53 & 1.18 & 5.44 & * & 155 & $36.1 \%$ & 0.60 & 0.18 & 1.98 & \\
\hline $50-69$ years & 366 & $71.0 \%$ & 6.14 & 2.88 & 13.09 & $* * *$ & 271 & $35.8 \%$ & 0.60 & 0.21 & 1.73 & \\
\hline Highest education & 756 & & & & & & 449 & & & & & \\
\hline None (ref) & 124 & $64.4 \%$ & & & & & 85 & $27.1 \%$ & & & & \\
\hline Primary & 284 & $62.5 \%$ & 0.92 & 0.54 & 1.56 & & 183 & $37.3 \%$ & 1.60 & 0.80 & 3.19 & \\
\hline Secondary & 239 & $46.9 \%$ & 0.49 & 0.26 & 0.93 & $*$ & 117 & $41.4 \%$ & 1.90 & 0.82 & 4.43 & \\
\hline Tertiary or higher & 109 & $50.5 \%$ & 0.56 & 0.28 & 1.12 & & 64 & $37.4 \%$ & 1.61 & 0.60 & 4.28 & \\
\hline Residence type & 756 & & & & & & 449 & & & & & \\
\hline Urban (ref) & 132 & $51.2 \%$ & & & & & 77 & $46.5 \%$ & & & & \\
\hline Rural & 624 & $55.7 \%$ & 1.20 & 0.70 & 2.06 & & 372 & $34.1 \%$ & 0.60 & 0.28 & 1.26 & \\
\hline Current smoking & 756 & & & & & & 449 & & & & & \\
\hline No (ref) & 682 & $56.8 \%$ & & & & & 415 & $37.9 \%$ & & & & \\
\hline Yes & 74 & $45.1 \%$ & 0.62 & 0.30 & 1.28 & & 34 & $33.1 \%$ & 0.81 & 0.26 & 2.53 & \\
\hline Binge alcohol use & 756 & & & & & & 449 & & & & & \\
\hline No (ref) & 680 & $60.0 \%$ & & & & & 420 & $37.6 \%$ & & & & \\
\hline Yes & 76 & $28.3 \%$ & 0.26 & 0.13 & 0.54 & $* * *$ & 29 & $32.2 \%$ & 0.79 & 0.24 & 2.60 & \\
\hline Adding table salt & 755 & & & & & & 448 & & & & & \\
\hline No (ref) & 483 & $56.5 \%$ & & & & & 295 & $33.7 \%$ & & & & \\
\hline Yes & 272 & $51.1 \%$ & 0.81 & 0.52 & 1.24 & & 153 & $43.7 \%$ & 1.53 & 0.85 & 2.74 & \\
\hline Body mass index & 745 & & & & & & 440 & & & & & \\
\hline Normal, BMI < 25 (ref) & 267 & $44.7 \%$ & & & & & 143 & $46.2 \%$ & & & & \\
\hline Overweight, BMI 25-30 & 229 & $53.6 \%$ & 1.43 & 0.84 & 2.43 & & 127 & $33.8 \%$ & 0.59 & 0.27 & 1.30 & \\
\hline Obese, BMI $>30$ & 249 & $66.8 \%$ & 2.48 & 1.36 & 4.53 & $* *$ & 170 & $28.0 \%$ & 0.45 & 0.21 & 0.96 & * \\
\hline Known diabetes & 756 & & & & & & 449 & & & & & \\
\hline No (ref) & 685 & $53.0 \%$ & & & & & 391 & $35.9 \%$ & & & & \\
\hline Yes & 71 & $69.4 \%$ & 2.01 & 0.86 & 4.68 & & 58 & $46.2 \%$ & 1.54 & 0.61 & 3.85 & \\
\hline Other comorbidities & 756 & & & & & & 449 & $* * *$ & & & & \\
\hline No (ref) & 621 & $54.4 \%$ & & & & & 368 & $35.5 \%$ & & & & \\
\hline Yes & 135 & $55.2 \%$ & 1.03 & 0.57 & 1.85 & & 81 & $43.2 \%$ & 1.38 & 0.73 & 2.62 & \\
\hline Lifestyle advice & 756 & & & & & & 449 & & & & & \\
\hline No (ref) & 95 & $23.5 \%$ & & & & & 30 & $59.2 \%$ & & & & \\
\hline Yes & 661 & $59.1 \%$ & 4.71 & 2.26 & 9.81 & $* * *$ & 419 & $35.8 \%$ & 0.39 & 0.14 & 1.07 & \\
\hline
\end{tabular}

${ }^{*} p<0.05,{ }^{* *} p<0.001$, and ${ }^{* * *} p<0.0001$. Subtotals with missing values are indicated in italics. Binge use of alcohol was defined as drinking six or more units of alcohol in one occasion during the preceding 30 days. Added salt at meals was defined as a response other than "never" in response to the question, "how often do you add salt or salty sauce to your food just before or during eating?" Other comorbidities: self-reported history asthma, cancer, renal disease, "depression or other mental illnesses," or HIV. Participants were considered to have received lifestyle advice if they responded yes to any of the options for the question, "during the past three years, has a doctor or any other health worker advised you to do any of the following, quit using tobacco, reduce salt, eat at least five servings of fruit and/or vegetables each day, start or do more physical activity?"

early 2017, which revealed that service inputs such as personnel, essential medicines for CV risk management (with the exception of statins), and equipment were generally available at the facility level. Key gaps identified included provider knowledge on hypertension and other chronic NCDs and lack of recall systems for longitudinal follow-up of patients [28].

The authors recognize that five years have elapsed since data collection and that updated assessment of the prevalence of hypertension and its control (and service delivery processes related to control) is due. This could be achieved by incorporating NCDs data elements in other national, population-based surveys that may occur before the next STEPS survey, such as the triennial Botswana AIDS Impact Survey. Our reported analysis would provide a reference baseline against which future analyses and impact evaluation of the 2016 Primary Healthcare guidelines can be compared.

The fact that two-thirds of treated hypertensives had $\mathrm{BP} \geq 140 / 90$ (a liberal threshold given evidence that lower BP targets confer cardiovascular benefit), and obese individuals were less likely to achieve control, speaks to the need of couple treatment with strong behavioral supports for medication and healthy lifestyle adherence. The paradoxical association of lifestyle advice with lower likelihood of control in our analysis suggests that this advice, typically delivered as brief mention by providers not trained in motivational counseling, may be a marker of disease that is "difficult" to manage. There is a need to scale up effective approaches for hypertension management adherence support in resource-limited settings. One such approach, not 


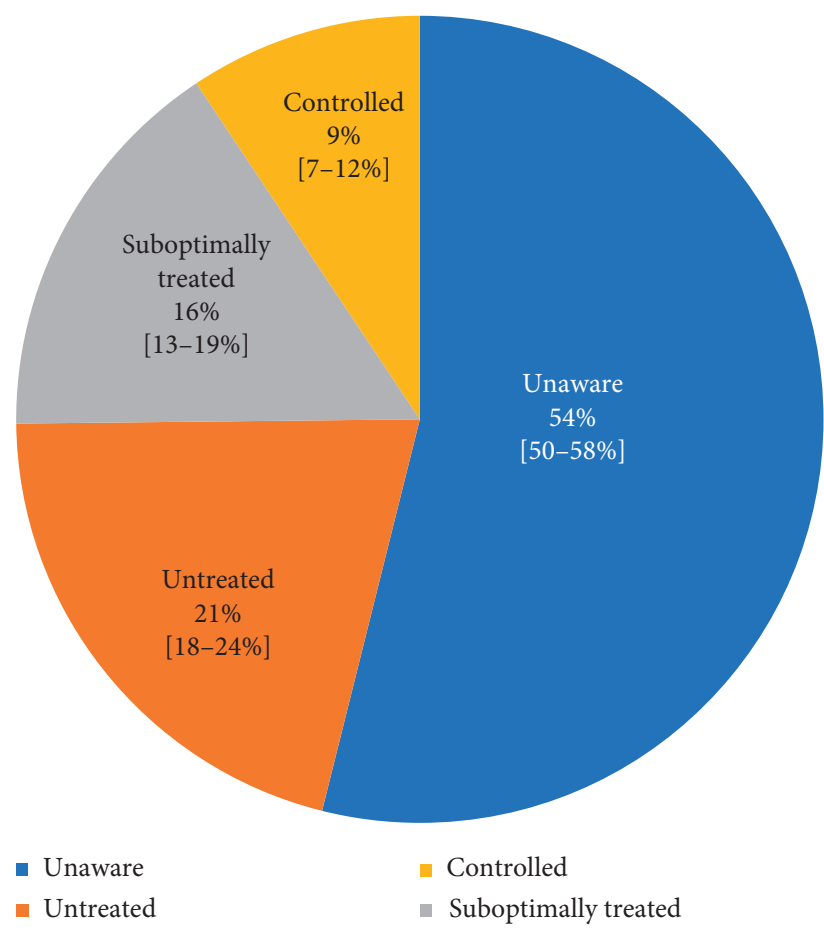

FIgURE 3: Age-standardized proportions of undiagnosed, untreated, and suboptimally controlled blood pressure among adult hypertensives. The common denominator used here was the number of participants with hypertension, defined as self-report of being on antihypertensives in the last two weeks (treated) or having elevated blood pressure (BP) based on survey measurement (mean $\mathrm{SBP} \geq 140 \mathrm{mmHg}$ or mean $\mathrm{DBP} \geq 90 \mathrm{mmHg}$ ). Hypertensives were divided into mutually exclusive groups. Unaware hypertensives were those who had elevated BP, yet had not previously been informed of a hypertension diagnosis by a health professional. Untreated hypertensives were those who had been previously informed of a hypertension diagnosis but were not on medications in the past 2 weeks and had elevated BP. Suboptimally treated hypertensives were those who had been previously informed of a diagnosis and were on medications, yet had elevated BP. Controlled hypertensives were those who had been previously informed of diagnosis, were on medications and had BP $<140 / 90$.

currently available in routine care in Botswana and many developing countries, is home-based BP monitoring and motivational counseling delivered by trained lay health workers supervised by health professionals. This has been demonstrated to be effective in influencing behavior and reducing $\mathrm{BP}$ in cluster randomized controlled trials in Argentina [29], Malaysia, and Colombia [30].

With regard to correlates of hypertension care cascade phases, being older, obese, and having diabetes or other comorbidity were all associated with high likelihood of hypertension diagnosis. This is likely due to more interaction with healthcare services of these individuals rendering them more accepting of and available for opportunistic screening. There may also be a contribution of more proactive opportunistic screening by providers who appropriately assess these individuals at higher cardiovascular risk. We also found that hypertensive men were less likely to be aware or controlled than women, a phenomenon described in many other studies [21]. Our findings support the hypothesis that diabetes, as a chronic condition for which regular interaction with healthcare is necessary, provides a gateway for opportunistic screening for hypertension and linkage to treatment. As such it is conceivable that this would be the case for other chronic conditions including HIV, and indeed studies have found higher hypertension awareness [31], treatment [31], and control [32] among HIV-infected individuals. These findings support an approach of integrating hypertension service delivery into existing longitudinal care platforms for HIV and other chronic conditions in order to enhance hypertension detection and management, an approach that has been recommended widely $[33,34]$.

While our unadjusted analyses found associations between education and hypertension outcomes similarly to some studies [35-37], these associations were not statistically significant in our multivariable analysis. Exploratory analyses did not identify confounding effects of variables not included in some studies: rural residence, comorbidities, and lifestyle advice. In addition to contribution of insufficient power (supported by our 95\% confidence Intervals), context factors may be at play. This would be consistent with studies reporting differing relationships between education and hypertension treatment outcomes [38] and a systematic review on the determinants of hypertension noting that the association between education and hypertension is among those inconsistently reported [39].

4.1. Limitations. Our study has several notable limitations. The definition of hypertension used in this study excluded individuals who had been informed of hypertension but were not on medications and did not have elevated BP and thus may have underestimated hypertension prevalence. The definition we used was preferred as it yields more conservative estimates and has been used in many other studies [10,19-21], with which comparisons can be made. As we used the mean for all three BP measurements, there is a possibility that "white coat hypertension" may have falsely raised our estimates for hypertension prevalence. Sensitivity analyses (using only BP values from the $2^{\text {nd }}$ and $3^{\text {rd }}$ measurements) were reassuring in this regard, with findings similar to our primary results. The STEPS survey relied on self-report of antihypertensive treatment which may have resulted in misclassification and underestimation of hypertension prevalence and proportion treated. Only $64 \%$ of target sample size was achieved (enrollment was lower in remote, sparsely populated areas with challenging terrain such that survey staff were unable to reach communities), and only a third of the sample was male (males tended to not be home during survey visit times which included early evenings), limiting the sample's representativeness of Botswana's population. Nonetheless, our hypertension prevalence estimates were comparable to those from other studies in the region. The survey, whose primary objective was to estimate prevalence of NCD risk factors, did not include data on some factors known to be associated with hypertension control such as medication adherence, provider training, care facility type, and 
TABLE 5: Multivariable logistic regression of factors associated with hypertension, awareness, treatment, and control among adults in Botswana.

\begin{tabular}{|c|c|c|c|c|c|c|c|c|c|c|c|c|c|c|c|c|}
\hline & \multicolumn{4}{|c|}{ Hypertension $(n=1,393)$} & \multicolumn{4}{|c|}{$\begin{array}{c}\text { Awareness, among } \\
\text { hypertensives }(n=756)\end{array}$} & \multicolumn{4}{|c|}{$\begin{array}{l}\text { Treatment, among } \\
\text { aware hypertensives } \\
\quad(n=449)\end{array}$} & \multicolumn{4}{|c|}{$\begin{array}{l}\text { Control, among treated } \\
\text { hypertensives }(n=171)\end{array}$} \\
\hline & $\mathrm{aOR}$ & $95 \%$ & $\mathrm{CI}$ & $p$ & $\mathrm{aOR}$ & $95 \%$ & $\mathrm{CI}$ & $p$ & $\mathrm{aOR}$ & $95 \%$ & $\mathrm{CI}$ & $p$ & $\mathrm{aOR}$ & $95 \%$ & $\mathrm{CI}$ & $p$ \\
\hline Male & 1.30 & 1.03 & 1.65 & * & 0.62 & 0.41 & 0.94 & $*$ & 0.91 & 0.50 & 1.69 & & 0.36 & 0.16 & 0.83 & $*$ \\
\hline Age & 1.04 & 1.03 & 1.06 & $* * *$ & 1.05 & 1.03 & 1.06 & $* * *$ & 1.07 & 1.04 & 1.09 & $* * *$ & 1.00 & 0.97 & 1.03 & \\
\hline Secondary school or higher & 0.86 & 0.66 & 1.12 & & 1.46 & 0.95 & 2.26 & & 1.49 & 0.80 & 2.77 & & 1.34 & 0.56 & 3.23 & \\
\hline Rural residence & 1.09 & 0.84 & 1.40 & & 0.99 & 0.64 & 1.54 & & 1.07 & 0.61 & 1.88 & & 0.74 & 0.36 & 1.51 & \\
\hline Current smoking & 1.40 & 0.98 & 1.99 & & 0.93 & 0.5 & 1.73 & & 1.65 & 0.69 & 3.91 & & 1.75 & 0.56 & 5.44 & \\
\hline Binge alcohol use & 1.21 & 0.8 & 1.81 & & 1.28 & 0.73 & 2.25 & & 0.41 & 0.18 & 0.94 & $*$ & 0.99 & 0.22 & 4.41 & \\
\hline Adding table salt & 0.80 & 0.63 & 1.01 & & 0.71 & 0.50 & 1.00 & & 0.95 & 0.61 & 1.49 & & 1.78 & 1.01 & 3.16 & \\
\hline Low fruit/vegetable intake & 0.77 & 0.44 & 1.38 & & 0.99 & 0.38 & 2.53 & & 1.24 & 0.48 & 3.20 & & 0.47 & 0.15 & 1.50 & \\
\hline \multicolumn{17}{|l|}{$\mathrm{BMI}<25$ (ref) } \\
\hline BMI 25-30 & 1.56 & 1.20 & 2.04 & $* *$ & 1.66 & 1.05 & 2.65 & $*$ & 0.94 & 0.52 & 1.68 & & 0.70 & 0.32 & 1.56 & \\
\hline $\mathrm{BMI}>30$ & 2.96 & 2.16 & 4.04 & $* * *$ & 2.27 & 1.40 & 3.67 & $* *$ & 2.17 & 1.12 & 4.22 & $*$ & 0.32 & 0.15 & 0.66 & $* *$ \\
\hline Known diabetes & 4.00 & 1.86 & 8.59 & $* * *$ & 3.30 & 1.44 & 7.55 & $* *$ & 1.62 & 0.74 & 3.56 & & 1.96 & 0.81 & 4.74 & \\
\hline Other comorbidities & 0.93 & 0.66 & 1.31 & & 1.85 & 1.06 & 3.23 & $*$ & 1.15 & 0.67 & 1.97 & & 1.59 & 0.87 & 2.89 & \\
\hline Lifestyle advice & N/A & N/A & N/A & & N/A & N/A & N/A & & 4.98 & 2.42 & 10.2 & $* * *$ & 0.27 & 0.08 & 0.83 & * \\
\hline
\end{tabular}

${ }^{*} p<0.05,{ }^{* *} p<0.001$, and ${ }^{* * *} p<0.0001$. BMI = body mass index. Binge use of alcohol was defined as drinking six or more units of alcohol in one occasion during the preceding 30 days. Added salt at meals was defined as a response other than "never" in response to the question, "how often do you add salt or salty sauce to your food just before or during eating?" Other comorbidities: self-reported history asthma, cancer, renal disease, "depression or other mental illnesses," or HIV. Participants were considered to have received lifestyle advice if they responded yes to any of the options for the question, "during the past three years, has a doctor or any other health worker advised you to do any of the following, quit using tobacco, reduce salt, eat at least five servings of fruit and/or vegetables each day, start or do more physical activity?" The fully adjusted models included all variables listed in the table for a given hypertension status outcome.

healthcare utilization. The sample size of treated hypertensives was small such that analysis of correlates of control was insufficiently powered. That said, we were able to examine sociodemographic, behavioral, and clinical factors and document associations that may influence further research and practice.

\section{Conclusions}

We report the first nationally representative estimates of hypertension care cascade in Botswana, which will inform planning and serve as a baseline for future evaluations of national policy. Furthermore, we contribute to the relatively sparse evidence in the African setting on determinants of hypertension control performance, examining less frequently reported correlates. We found that nearly a third of the adult population was hypertensive and that there were large gaps in each care cascade step such that only $9 \%$ of all hypertensives were controlled, and a fifth of hypertensives who were diagnosed but untreated were in hypertensive crisis. Male and obese hypertensives were less likely to be controlled, while comorbidities such as diabetes may serve as a gateway to enhance hypertension awareness and treatment. These results highlight the need to accelerate hypertension control efforts in similar settings and may inform development of innovations that improve quality and effectiveness of care and ultimately reduce CVD-related deaths.

\section{Data Availability}

The data used to support the findings of this study were from the Botswana National NCD Survey conducted in 2014. Requests for survey data can be made to the Botswana Ministry of Health and Wellness.

\section{Conflicts of Interest}

The authors declare that they have no conflicts of interest.

\section{Authors' Contributions}

NT designed the study, conducted primary analysis, and drafted the manuscript. LC supported analysis and interpretation. DJH contributed to study design, analytic approach, interpretation, and manuscript drafting. MG contributed to data acquisition. All authors, including TM, GT, VL, MM, TM, JM, and SL, contributed to analysis interpretation, manuscript review, and revision.

\section{Acknowledgments}

The authors are grateful to the participants of this survey. The authors would also like to thank the Botswana Ministry of Health and Wellness' NCD Program, the World Health Organization-Botswana country office, and the World Health Organization HQ NCD Surveillance team for conducting the survey. Dissemination of this study is supported by the Peo Project (Planning for Southern African regional Center Research Excellence in NCDs), funded by The US National Institutes of Health/National Cancer Institute (Grant no. 5P20CA210283).

\section{References}

[1] WHO, WHO Global Status Report on NCDs 2014, WHO, Geneva, Switzerland, 2014, http://www.who.int/chp/ knowledge/en/. 
[2] C. M. M. Lawes, S. V. Hoorn, A. Rodgers, and I. Society, "Global burden of blood-pressure-related disease, 2001," The Lancet, vol. 371, pp. 1513-1518, 2008.

[3] M. E. Kruk, A. D. Gage, C. Arsenault et al., "High-quality health systems in the Sustainable Development Goals era: time for a revolution," The Lancet Global Health, vol. 6, no. 11, pp. e1196-e1252, 2018.

[4] J. E. Bennett, G. A. Stevens, C. D. Mathers et al., "NCD Countdown 2030: worldwide trends in non-communicable disease mortality and progress towards Sustainable Development Goal target 3.4," The Lancet, vol. 392, no. 10152, pp. 1072-1088, 2018.

[5] D. Ettehad, C. A. Emdin, A. Kiran et al., "Blood pressure lowering for prevention of cardiovascular disease and death: a systematic review and meta-analysis," The Lancet, vol. 387, no. 10022, pp. 957-967, 2016.

[6] C. K. Chow, "Prevalence, awareness, treatment, and control of hypertension in rural and urban communities in high-, middle-, and low-income countries," JAMA, vol. 310, no. 9, p. 959, 2013.

[7] V. J. Wirtz, W. A. Kaplan, G. F. Kwan, and R. O. Laing, "Access to medications for cardiovascular diseases in low- and middle-income countries," Circulation, vol. 133, no. 21, pp. 2076-2085, 2016.

[8] D. J. Hyman and V. N. Pavlik, "Characteristics of patients with uncontrolled hypertension in the United States," New England Journal of Medicine, vol. 345, no. 7, pp. 479-486, 2001.

[9] G. Sarganas and H. K. Neuhauser, "Untreated, uncontrolled, and apparent resistant hypertension: results of the German health examination survey 2008-2011," The Journal of Clinical Hypertension, vol. 18, no. 11, pp. 1146-1154, 2016.

[10] F. Ataklte, S. Erqou, S. Kaptoge, B. Taye, J. B. EchouffoTcheugui, and A. P. Kengne, "Burden of undiagnosed hypertension in sub-saharan Africa," Hypertension, vol. 65, no. 2, pp. 291-298, 2015.

[11] T. Beaney, A. E. Schutte, M. Tomaszewski et al., "May Measurement Month 2017: an analysis of blood pressure screening results worldwide," The Lancet Global Health, vol. 6, no. 7, pp. e736-e743, 2018.

[12] G. Musinguzi and F. Nuwaha, "Prevalence, awareness and control of hypertension in Uganda," PLoS One, vol. 8, no. 4, Article ID e62236, 2013.

[13] A. Dzudie, A. P. Kengne, W. F. T. Muna et al., "Prevalence, awareness, treatment and control of hypertension in a selfselected sub-Saharan African urban population: a cross-sectional study," BMJ Open, vol. 2, no. 4, p. e001217, 2012.

[14] A. Damasceno, A. Azevedo, C. Silva-Matos, A. Prista, D. Diogo, and N. Lunet, "Hypertension prevalence, awareness, treatment, and control in Mozambique," Hypertension, vol. 54, no. 1, pp. 77-83, 2009.

[15] B. M. Mayosi, A. J. Flisher, U. G. Lalloo, F. Sitas, S. M. Tollman, and D. Bradshaw, "The burden of noncommunicable diseases in South Africa," The Lancet, vol. 374, no. 9693, pp. 934-947, 2009.

[16] M. E. Kruk, G. Nigenda, and F. M. Knaul, "Redesigning primary care to tackle the global epidemic of noncommunicable disease," American Journal of Public Health, vol. 105, no. 3, pp. 431-437, 2015.

[17] World Health Organization, The WHO STEPwise Approach to Noncommunicable Diseases Risk Factor Surveillance, World Health Organization, Geneva, Switzerland, 2017, https://www. who.int/ncds/surveillance/steps/STEPS_Manual.pdf?ua=1.

[18] R. Asmar, J. Khabouth, J. Topouchian, R. El Feghali, and J. Mattar, "Validation of three automatic devices for self- measurement of blood pressure according to the international protocol: the Omron M3 intellisense (HEM-7051-E), the Omron M2 compact (HEM 7102-E), and the Omron R3-I plus (HEM 6022-E)," Blood Pressure Monitoring, vol. 15, no. 1, pp. 49-54, 2010.

[19] K. M. Berry, W.-a. Parker, Z. J. Mchiza et al., "Quantifying unmet need for hypertension care in South Africa through a care cascade: evidence from the SANHANES, 2011-2012," BMJ Global Health, vol. 2, no. 3, p. e000348, 2017.

[20] G. Wozniak, T. Khan, C. Gillespie et al., "Hypertension control cascade: a framework to improve hypertension awareness, treatment, and control," The Journal of Clinical Hypertension, vol. 18, no. 3, pp. 232-239, 2016.

[21] P. Geldsetzer, J. Manne-Goehler, M.-E. Marcus et al., "The state of hypertension care in 44 low-income and middleincome countries: a cross-sectional study of nationally representative individual-level data from 1.1 million adults," The Lancet, vol. 394, no. 10199, pp. 652-662, 2019.

[22] A. V. Chobanian, G. L. Bakris, H. R. Black et al., "The Seventh report of the Joint national committee on prevention, detection, evaluation, and treatment of high blood pressure," JAMA, vol. 289, no. 19, pp. 2560-2572, 2003.

[23] F. S. Sarfo, L. M. Mobula, G. Burnham et al., "Factors associated with uncontrolled blood pressure among Ghanaians : evidence from a multicenter hospital-based study," PLoS One, vol. 13, 2018.

[24] S. F. Mohamed, M. K. Mutua, R. Wamai et al., "Prevalence, awareness, treatment and control of hypertension and their determinants: results from a national survey in Kenya," BMC Public Health, vol. 18, no. 3, 2018.

[25] Ministry of Health, The Essential Health Service Package for Botswana, Government of Botswana, Gaborone, Botswana, 2010.

[26] P. K. Whelton, R. M. Carey, W. S. Aronow et al., "Response to letter to editor "2017 ACC/AHA/AAPA/ABC/ACPM/AGS/ $\mathrm{APhA} / \mathrm{ASH} / \mathrm{ASPC} / \mathrm{NMA} / \mathrm{PCNA}$ guideline for the prevention, detection, evaluation, and management of high blood pressure in adults", Journal of the American Society of Hypertension, vol. 12, 2018.

[27] M. E. Kruk, A. D. Gage, N. T. Joseph, G. Danaei, S. GarcíaSaisó, and J. A. Salomon, "Mortality due to low-quality health systems in the universal health coverage era: a systematic analysis of amenable deaths in 137 countries," The Lancet, vol. 392, 2018.

[28] N. M. Tapela, G. Tshisimogo, B. P. Shatera et al., "Integrating noncommunicable disease services into primary health care, Botswana," Bulletin of the World Health Organization, vol. 97, no. 2, pp. 142-153, 2019.

[29] J. He, V. Irazola, K. T. Mills et al., "Effect of a community health worker-led multicomponent intervention on blood pressure control in low-income patients in Argentina: a randomized clinical trial," JAMA, vol. 318 , no. 11 , pp. 1016-1025, 2017.

[30] J.-D. Schwalm, T. McCready, P. Lopez-Jaramillo et al., "A community-based comprehensive intervention to reduce cardiovascular risk in hypertension (HOPE 4): a clusterrandomised controlled trial," The Lancet, vol. 394, no. 10205, pp. 1231-1242, 2019.

[31] J. Manne-goehler, M. J. Siedner, L. Montana et al., "Hypertension and diabetes control along the hiv care cascade in rural South Africa," Journal of the International AIDS Society, vol. 22, pp. 1-5, 2019.

[32] D. Kwarisiima, M. Atukunda, A. Owaraganise et al., "Hypertension control in integrated hiv and chronic disease 
clinics in Uganda in the search study," BMC Public Health, vol. 19, pp. 1-10, 2019.

[33] V. Haldane, H. Legido-Quigley, F. L. H. Chuah et al., "Integrating cardiovascular diseases, hypertension, and diabetes with HIV services: a systematic review," AIDS Care, vol. 30, no. 1, pp. 103-115, 2018.

[34] M. Rabkin, A. Palma, M. L. McNairy et al., "Integrating cardiovascular disease risk factor screening into HIV services in Swaziland," AIDS, vol. 32, pp. S43-S46, 2018.

[35] L. J. Ware, G. Chidumwa, K. Charlton, A. E. Schutte, and P. Kowal, "Predictors of hypertension awareness, treatment and control in South Africa: results from the WHO-SAGE population survey (Wave 2)," Journal of Human Hypertension, vol. 33, no. 2, pp. 157-166, 2019.

[36] L. Yang, J. Yan, X. Tang, X. Xu, W. Yu, and H. Wu, "Prevalence, awareness, treatment, control and risk factors associated with hypertension among adults in southern China, 2013," PLoS One, vol. 11, no. 1, 2016.

[37] T. D. Chiara, A. Scaglione, S. Corrao, C. Argano, A. Pinto, and R. Scaglione, "Association between low education and higher global cardiovascular risk," The Journal of Clinical Hypertension, vol. 17, no. 5, pp. 332-337, 2015.

[38] P. Lloyd-sherlock, J. Beard, N. Minicuci, S. Ebrahim, and S. Chatterji, "Hypertension among older adults in low- and middle-income countries: prevalence, awareness and control," International Journal of Epidemiology, vol. 43, no. 1, pp. 116-128, 2014.

[39] W. K. Bosu, J. Moses, K. Aheto, E. Zucchelli, and S. T. Reilly, "Determinants of systemic hypertension in older adults in Africa : a systematic review," BMC Cardiovascular Disorders, vol. 19, 2019. 